The Astrophysical Journal, 337:959-963, 1989 February 15

(C) 1989. The American Astronomical Society. All rights reserved. Printed in U.S.A.

\title{
ISOTOPE ABUNDANCES OF SOLAR CORONAL MATERIAL DERIVED FROM SOLAR ENERGETIC PARTICLE MEASUREMENTS
}

\author{
R. A. Mewaldt and E. C. Stone \\ California Institute of Technology \\ Received 1988 July 11 ; accepted 1988 August 8
}

\begin{abstract}
Coronal isotopic abundances for the elements $\mathrm{He}, \mathrm{C}, \mathrm{N}, \mathrm{O}, \mathrm{Ne}$, and $\mathrm{Mg}$ are derived from previously published measurements of the isotopic composition of solar energetic particles by first measuring, and then correcting for, the charge-to-mass-dependent fractionation due to solar flare acceleration and propagation processes. The resulting coronal composition generally agrees with that of other samples of solar system material, but the previously noted difference between the solar flare and solar wind ${ }^{22} \mathrm{Ne} /{ }^{20} \mathrm{Ne}$ ratios remains unresolved.
\end{abstract}

Subject headings: cosmic rays: abundances - Sun: abundances — Sun: corona

\section{INTRODUCTION}

Although the Sun contains more than $99 \%$ of the solar system material, most of our present knowledge of the solar system element and isotope distribution comes from studies of terrestrial, meteoritic, and lunar material. Spectroscopic studies of the solar composition are subject to a number of sources of uncertainty, and in the case of isotopic abundances are available for only a few elements. Measurements of solar energetic particles (SEPs) accelerated during large solar flares provide a means of sampling directly the composition of solar material and thereby determining its composition. However, in interpreting such measurements, there has always been a question of the extent to which the observed particle composition might have been fractionated, either during the acceleration process itself or during the particles' subsequent propagation through interplanetary space.

Recently, a Voyager study by Breneman and Stone (1985, hereafter $B \& S$ ) provided a quantitative answer to the fractionation question. By combining elemental composition measurements from 10 large solar energetic particle events observed by Voyager 1 and 2 with ionic charge-state measurements made simultaneously in the ISEE 3 (ICE) spacecraft (Luhn et al. 1984), B\&S showed that the ionic charge-to-mass ratio $(Q / M)$ is the principal organizing factor for the fractionation of SEP elemental abundances by acceleration and propagation processes, and for flare-to-flare composition variations. They found that such composition variations are a smooth function of $Q / M$ that is well described by a power law. By correcting for these fractionation effects, $B \& S$ derived unfractionated coronal abundances for 20 elements. In this paper we report on an extension of the B\&S approach to SEP isotope measurements, using SEP isotope results reported previously from ISEE 3 and IMP 8.

\section{APPROACH}

Most of the measurements used for this study were obtained by the Caltech Heavy Isotope Spectrometer (HIST) on ISEE 3 during the large solar flare of 1978 September 23. When these measurements were originally published (Mewaldt, Spalding, and Stone 1984 and references therein), the relative isotope abundances were reported essentially as observed, with corrections for small differences in the energy-per-nucleon intervals covered by the observations of each isotope, but with no corrections for any possible fractionation due to acceleration or propagation effects. To determine the magnitude and $Q / M$ dependence of the fractionation in the 1978 September 23 flare event, we make use of the charge-state measurements made during this flare on the same spacecraft by the Max-Planck/ Maryland group (Luhn et al. 1984), and of the elemental composition measured by our own experiment (Mewaldt, Spalding, and Stone 1984). Figure 1 plots the ratio of the abundances measured in this flare event to the coronal abundances derived by $\mathrm{B} \& \mathrm{~S}$, as a function of $Q / M$. Note that in this flare elements that retain several electrons, such as $\mathrm{Fe}, \mathrm{Si}$, and $\mathrm{S}$, are depleted relative to those elements that are nearly fully stripped, such as C. As in the B\&S study, this fractionation is well described by the function $(Q / M)^{\alpha}$, where in this case a least-squares fit gives $\alpha=1.95 \pm 0.44$. From this dependence it would also be expected that the abundance of heavier isotopes such as ${ }^{22} \mathrm{Ne}$ would be slightly depleted in this flare relative to that of lighter isotopes such as ${ }^{20} \mathrm{Ne}$.

To correct for the effects of fractionation in this flare and thereby obtain measurements of the coronal isotopic composition, we use

$$
\frac{S_{i}}{S_{j}}=\left[\frac{M_{i}}{M_{j}}\right]^{\alpha}\left[\frac{N_{i}}{N_{j}}\right],
$$

where $S_{i}$ and $S_{j}$ are the desired coronal abundances of isotopes $i$ and $j$ of a given element with masses $M_{i}, M_{j} ; N_{i}$ and $N_{j}$ are the observed abundances, as reported by Mewaldt, Spalding, and Stone (1984); and $\alpha=1.95$.

We have also considered the magnitude of fractionation effects on solar flare isotope results reported by others. To do this, we note from Figure 1 (see also Fig. 2 in Breneman and Stone 1985) that a reasonable estimate of the value of $\alpha$ for a particular flare can be obtained simply from $\left.[\mathrm{Fe} / \mathrm{O})_{f}\right]$, the $\mathrm{Fe}$ to $\mathrm{O}$ ratio of the flare measured in the same experiment. We then use

$$
\alpha_{\text {est }}=\frac{\ln \left[(\mathrm{Fe} / \mathrm{O})_{f} /(\mathrm{Fe} / \mathrm{O})_{c}\right]}{\left.\ln [Q / M)_{\mathrm{Fe}} /(Q / M)_{\mathrm{O}}\right]},
$$

where $(\mathrm{Fe} / \mathrm{O})_{c}=0.224 \pm 0.032$ is the coronal $\mathrm{Fe}$ to $\mathrm{O}$ ratio from B\&S. As an estimate of the charge states of $\mathrm{Fe}$ and $\mathrm{O}$, 


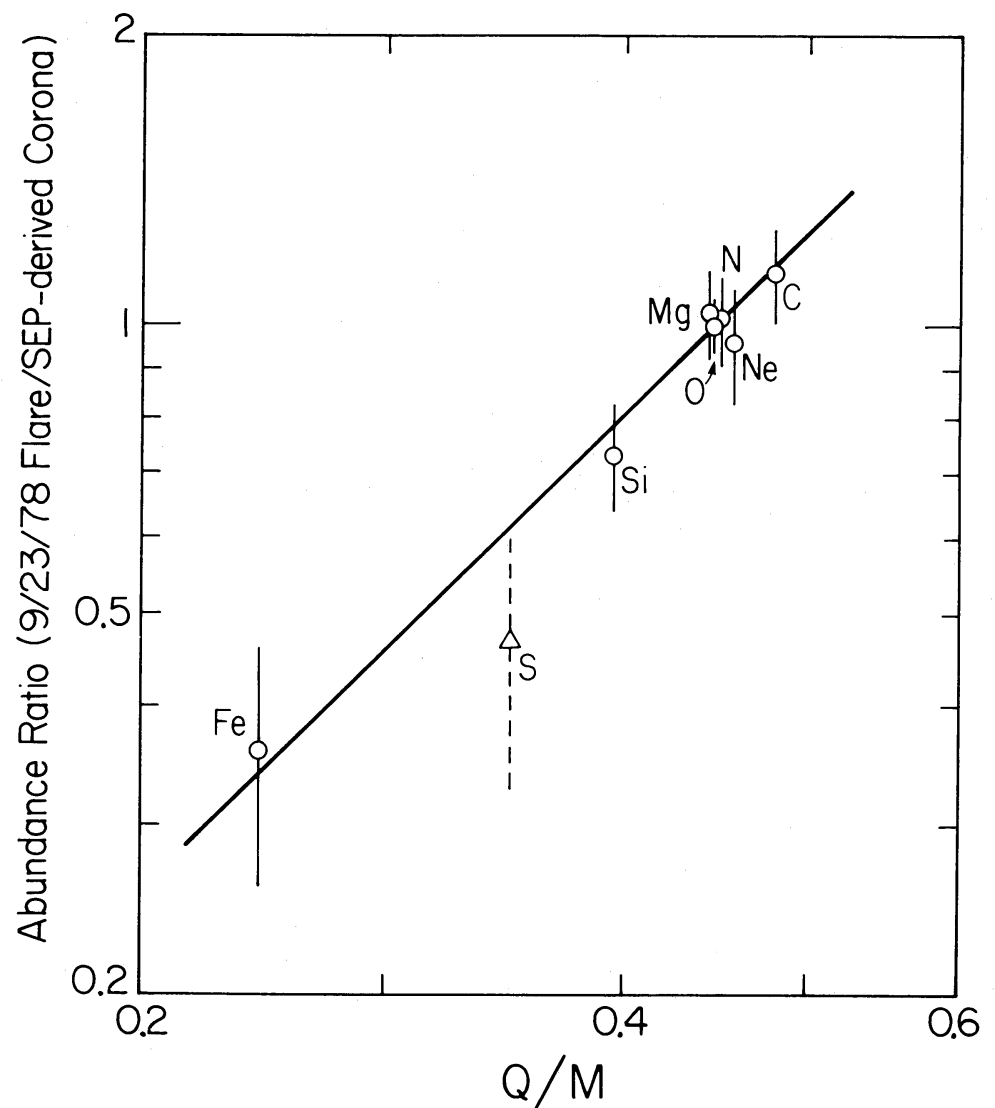

Fig. 1.-Ratio of the element abundance measured in the 1978 September 23 flare (Mewaldt, Spalding, and Stone 1984) to that for the SEP-derived corona (Breneman and Stone 1985), plotted as a function of the measured charge-to-mass ratio (Luhn et al. 1984). The solid line is a least-squares fit to $(Q / M)^{\alpha}$ giving $\alpha=1.95 \pm 0.44$. The $S$ point is based on measurements by McGuire, von Rosenvinge, and McDonald (1979) and was not included in the fit.

we use the average values $Q(\mathrm{Fe})=14.9 \pm 0.09$ and $Q(\mathrm{O})=$ $7.00 \pm 0.02$ from Luhn et al. (1984), noting that in large flares the observed charge states do not appear to vary significantly from flare to flare. We then use equation (1) to obtain an estimate of the coronal isotope abundances.

\section{RESULTS}

Table 1 includes both our measured isotope ratios from Mewaldt, Spalding, and Stone (1984) and the corresponding coronal abundance ratio obtained by correcting for the

TABLE 1

IsOTOPIC ABUNDANCES ObSERVED IN SEPS AND DEDUCED FOR THE CORONA

\begin{tabular}{cccl}
\hline \hline $\begin{array}{c}\text { Isotope } \\
\text { Ratio }\end{array}$ & $\begin{array}{c}\text { Measured Value } \\
\text { in SEPs }\end{array}$ & $\begin{array}{c}\text { Value Deduced } \\
\text { for the Corona }\end{array}$ & $\begin{array}{c}\text { Anders and } \\
\text { Ebihara 1982 }\end{array}$ \\
\hline${ }^{13} \mathrm{He} /{ }^{4} \mathrm{He} \ldots \ldots \ldots$. & $\leq 2.6 \times 10^{-3}$ & $\leq 1.9 \times 10^{-3}$ & $4.3 \times 10^{-4}$ \\
${ }^{13} \mathrm{C} /{ }^{12} \mathrm{C} \ldots \ldots \ldots$. & $0.0095_{-0.0029}^{+0.0042}$ & $0.0111_{-0.0034}^{+0.0049}$ & 0.0111 \\
${ }^{14} \mathrm{C} /{ }^{12} \mathrm{C} \ldots \ldots \ldots \ldots$ & $<0.0014$ & $<0.0019$ & 0.00 \\
${ }^{15} \mathrm{~N} /{ }^{14} \mathrm{~N} \ldots \ldots \ldots$. & $0.008_{-0.005}^{+0.010}$ & $0.009_{-0.006}^{+0.012}$ & 0.0037 \\
${ }^{17} \mathrm{O} /{ }^{16} \mathrm{O} \ldots \ldots \ldots$. & $\leq 0.0021$ & $\leq 0.0024$ & 0.00037 \\
${ }^{18} \mathrm{O} /{ }^{16} \mathrm{O} \ldots \ldots \ldots$. & $0.0015_{-0.0007}^{+0.0011}$ & $0.0019_{-0.0009}^{+0.0014}$ & 0.00204 \\
${ }^{21} \mathrm{Ne} /{ }^{20} \mathrm{Ne} \ldots \ldots$. & $\leq 0.014$ & $\leq 0.015$ & 0.0024 \\
${ }^{22} \mathrm{Ne} /{ }^{20} \mathrm{Ne} \ldots \ldots$. & $0.109_{-0.019}^{+0.026}$ & $0.131_{-0.024}^{+0.032}$ & 0.073 \\
${ }^{25} \mathrm{Mg} /{ }^{24} \mathrm{Mg} \ldots \ldots$. & $0.148_{-0.046}^{+0.046}$ & $0.160_{-0.028}^{+0.050}$ & 0.129 \\
${ }^{26} \mathrm{Mg} /{ }^{24} \mathrm{Mg} \ldots \ldots$. & $0.148_{-0.025}^{+0.043}$ & $0.173_{-0.030}^{+0.050}$ & 0.142 \\
\hline
\end{tabular}

${ }^{\text {a }}$ Mewaldt, Spalding, and Stone 1984. observed fractionation in the 1978 September 23 flare. The uncertainties in the quoted coronal abundance ratios include the uncertainty in determining the value of $\alpha$. In Figure 2 we compare our derived coronal composition measurements with the "solar system" abundances of Anders and Ebihara (1982) and with the solar wind measurements of Geiss et al. (1972). Also shown in Figure 2 are various flare-average measurements reported by the Chicago group (which they obtained from measurements summed over anywhere from 3 to 10 flares), which we have corrected for fractionation effects as described above.

\section{DISCUSSION}

Note in Figure 2 that the ${ }^{13} \mathrm{C} /{ }^{12} \mathrm{C},{ }^{15} \mathrm{~N} /{ }^{14} \mathrm{~N},{ }^{18} \mathrm{O} /{ }^{16} \mathrm{O}$, ${ }^{25} \mathrm{Mg} /{ }^{24} \mathrm{Mg}$, and ${ }^{26} \mathrm{Mg} /{ }^{24} \mathrm{Mg}$ ratios that we obtain for the corona are consistent with the Anders and Ebihara (1982) compilation, in agreement with our earlier conclusions (Mewaldt, Spalding, and Stone 1984), which were based on the abundances measured in interplanetary space, uncorrected for fractionation effects. This is not surprising, because the magnitude of the fractionation correction is not large (typically $10 \%-$ $20 \%$ ).

The isotope ${ }^{22} \mathrm{Ne}$ is of special interest because of the wide range of ${ }^{22} \mathrm{Ne} /{ }^{20} \mathrm{Ne}$ ratios observed in various samples of solar system material (see, e.g., Podosek 1978). The isotopic composition of neon in the Sun is a subject of controversy, with Anders and Ebihara (1982) adopting the solar wind (SW) value $\left({ }^{22} \mathrm{Ne} /{ }^{20} \mathrm{Ne}=0.073\right)$ as a standard, while Cameron (1982) 


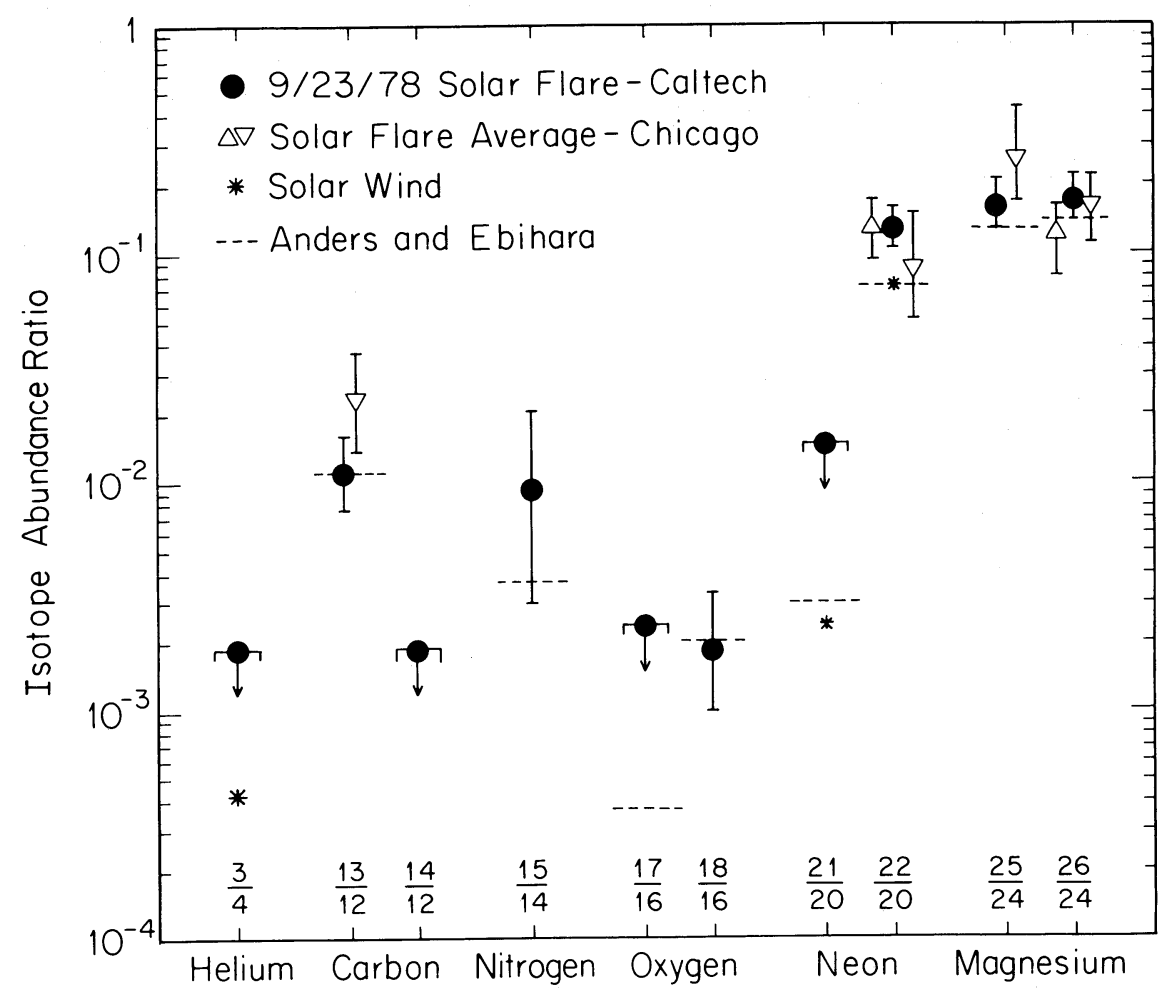

Fig. 2.-Comparison of isotopic abundances derived for the corona from SEP measurements with solar wind measurements (Geiss et al. 1972) and with the solar system abundances tabulated by Anders and Ebihara (1982). The SEP coronal abundances have been corrected for fractionation as described in the text, and are ( (1983) (open inverted triangles). The ${ }^{22} \mathrm{Ne} /{ }^{20} \mathrm{Ne}$ measurements of Dietrich and Simpson (1979) were corrected on a flare-by-flare basis using their reported Ne event totals and $\mathrm{Fe} / \mathrm{O}$ ratios. The correction for the $\mathrm{Mg}$ result of Dietrich and Simpson (1981) was derived from a weighted average of the $\mathrm{Fe} / \mathrm{O}$ ratios for flares included, while that for the results of Simpson, Wefel, and Zamow (1983) was derived from the average Fe/O ratio for their observation period.

adopts the meteoritic component "neon-A" $\left({ }^{22} \mathrm{Ne} /{ }^{20} \mathrm{Ne}\right.$ $=0.122$ ) as his standard for solar system neon. Figure 3 shows selected solar system measurements of ${ }^{22} \mathrm{Ne} /{ }^{20} \mathrm{Ne}$ on an expanded scale, including "neon-B," which is observed in both meteorites and lunar material and is believed to represent directly implanted solar wind. As concluded previously (Dietrich and Simpson 1979; Mewaldt et al. 1979; Mewaldt 1980; Mewaldt, Spalding, and Stone 1984), the spacecraft SEP measurements are inconsistent with the measured $\mathrm{SW}{ }^{22} \mathrm{Ne} /$ ${ }^{20} \mathrm{Ne}$ ratio. Note that the result of correcting our own SEP measurement for fractionation effects has increased the magnitude of this difference rather than narrowing it. It is interesting that there is excellent agreement between our derived ${ }^{22} \mathrm{Ne} /$ ${ }^{20} \mathrm{Ne}$ ratio and that derived from the Chicago measurements based on data summed over 10 large-flare events. This consistency suggests that our derived abundance of ${ }^{22} \mathrm{Ne}$ is typical, even though it is based on only a single large-flare event.

In Mewaldt, Spalding, and Stone (1984) we considered possible evidence for fractionation effects in SEPs by investigating the mass dependence of a "normalized abundance ratio," defined to be the measured isotope abundance ratio in SEPs divided by the corresponding "solar system" abundance ratio. Figure 4 shows the normalized abundance ratio for the 1978 September 23 flare as a function of the isotope mass ratio $\left(M_{j} / M_{i}\right)$, for both the Anders and Ebihara (1982) and the Cameron (1982) "solar system" tabulations. Also shown in Figure 4 is the mass dependence of the fractionation that we find for this flare. Note that for $\mathrm{C}, \mathrm{O}$, and $\mathrm{Mg}$ the derived fractionation is generally consistent with the normalized

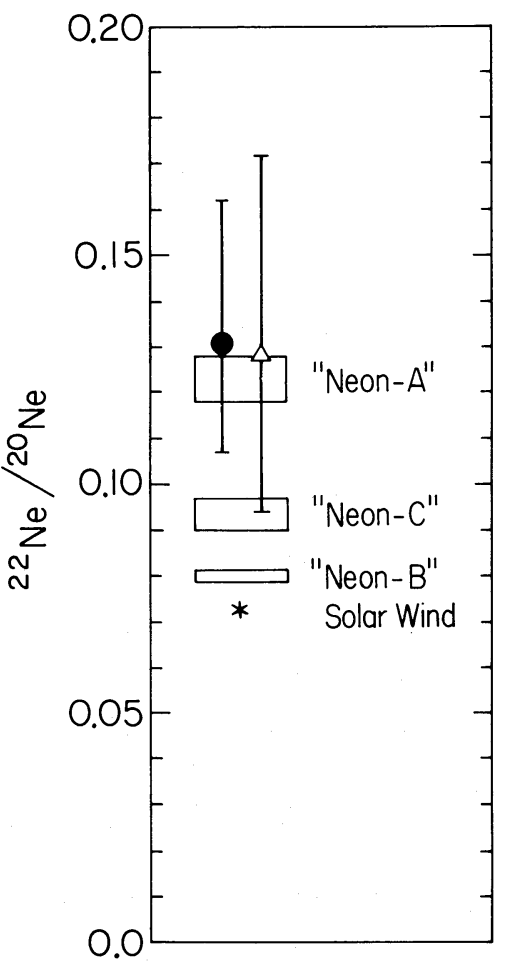

FIG. 3.-Comparison of selected solar system ${ }^{22} \mathrm{Ne} /{ }^{20} \mathrm{Ne}$ ratios. References to the points based on SEP and SW data are in the legend to Fig. 2, values for neon-A and neon-B are from Podosek (1978), and that for neon-C is from Black (1983). 


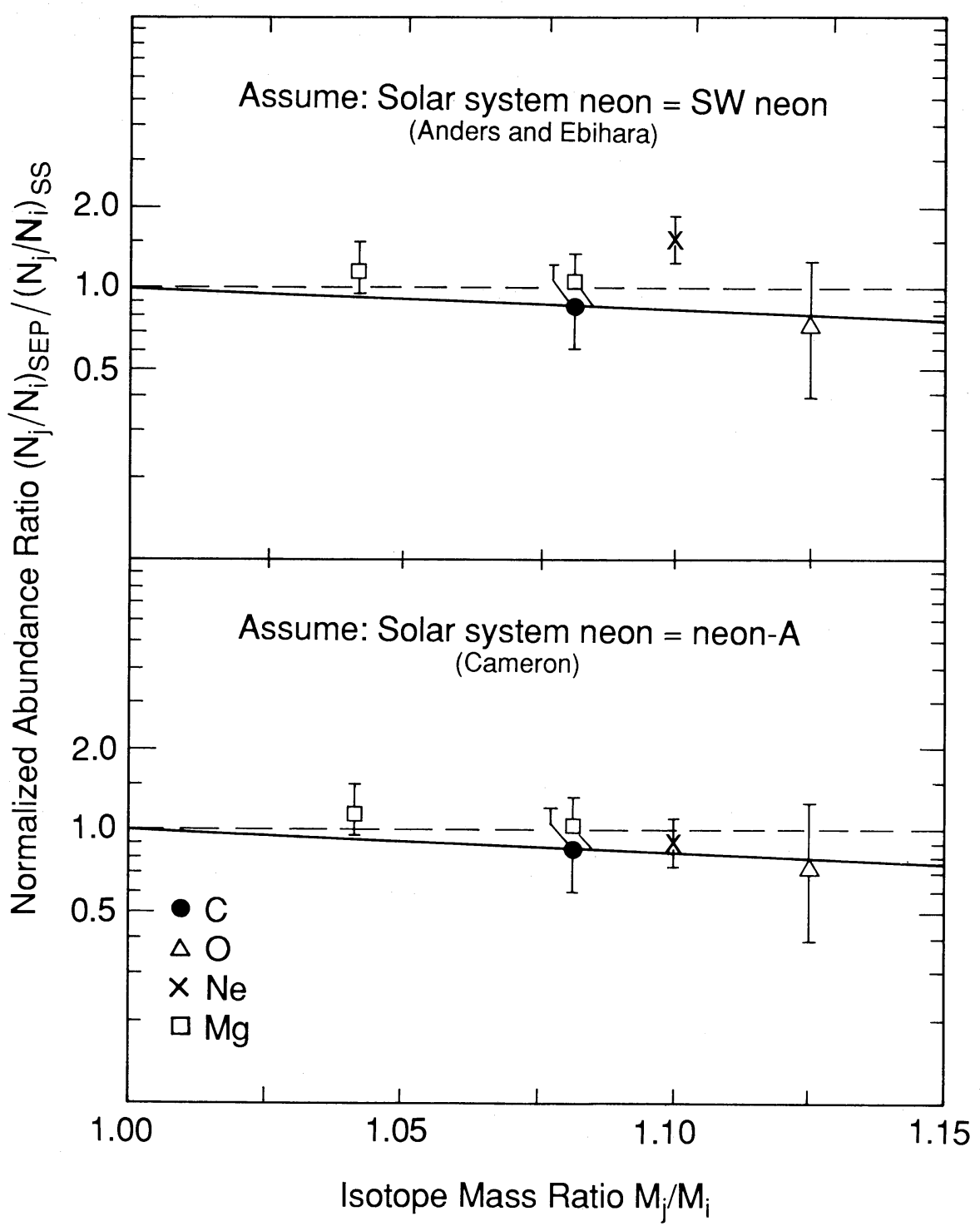

FIG. 4. - Plots of the "normalized abundance ratio" (measured value in SEPs divided by "solar system" value) vs. the isotope mass ratio for five pairs of isotopes. The top panel uses the Anders and Ebihara (1982) tabulation of solar system abundances (neon = SW neon), while the bottom panel uses the Cameron (1982) tabulation. The solid line in each panel represents the mass dependence of the fractionation that we find for the 1978 September 23 flare.

abundance ratios, as expected if the coronal and "solar system" isotopic abundances are the same. For ${ }^{22} \mathrm{Ne} /{ }^{20} \mathrm{Ne}$ there is consistency for the neon-A standard adopted by Cameron, but not with the solar wind standard adopted by Anders and Ebihara. This comparison does not necessarily imply that coronal neon is neon- $\mathrm{A}$, since there is a considerable range of possible ${ }^{22} \mathrm{Ne} /{ }^{20} \mathrm{Ne}$ ratios that are consistent with our SEP-derived coronal composition.

Figure 3 also includes the component "neon-C," which is observed in lunar material and has been interpreted to represent implanted SEP neon. (see, e.g., the review by Black 1983). Recent measurements (Wieler, Baur, and Signer 1986) confirm that there is a difference between neon- $\mathrm{C}$ and the measured $\mathrm{SW}{ }^{22} \mathrm{Ne} /{ }^{20} \mathrm{Ne}$ ratio, and also indicate a similar difference between SEP and SW Ar (Benkert et al. 1988; see also Black 1972). It should be pointed out that there should presumably also be a fractionation correction applied to the neon- $\mathrm{C}$ measurements. The magnitude of the correction in this case is difficult to determine directly because neon- $\mathrm{C}$ includes the integrated contributions of a large number of flares that occurred over millions of years. However, on the basis of measurements of contemporary flares at a similar energy $(\sim 1 \mathrm{MeV}$ nucleon $^{-1}$ ), the correction factor is probably not large. For example, in the energy interval from 1 to $4.6 \mathrm{MeV}^{\text {nucleon }}{ }^{-1}$ Mason et al. (1980) report an average $\mathrm{Fe} / \mathrm{O}$ ratio of 0.14 for the years 1973-1977, suggesting that the measured neon-C ratio should be multiplied by a correction factor of $\sim 1.1$, which would improve somewhat the agreement between the spacecraft and lunar measurements of solar flare neon isotopes.

In our first report of SEP ${ }^{22} \mathrm{Ne} /{ }^{20} \mathrm{Ne}$ observations (Mewaldt et al. 1979), we suggested that the difference between the measured SEP and SW isotope ratios could result from fractionation of either the SEP or SW abundances, both of which are presumably derived from coronal material. We have now measured and corrected for the fractionation in solar energetic particle events and find that the resulting value that we derive 
for ${ }^{22} \mathrm{Ne} /{ }^{20} \mathrm{Ne}$ in the solar corona differs from the measured SW value to an even greater extent, while we obtain quite reasonable values for the coronal isotopic composition of the other elements studied. Isotope fractionation during solar $\underset{1 \rightarrow 1}{ }$ wind acceleration remains as a possibility for explaining this difference.
We are grateful to R. E. Vogt and J. D. Spalding for their contributions to the HIST program. This work was supported in part by the National Aeronautics and Space Administration under grants NAG 5-722 and NGR 05-001-160.
Anders, E., and Ebihara, M. 1982, Geochim. Cosmochim. Acta, 46, 2363. Benkert, J.-P Baur, H Pedroni, A., Wieler, R., and Signer, P. 1988, in Lunar and Planetary Science XIX (Abstracts), Part 1 (Houston: Lunar and Planetary Science Institute and Universities Space Research Association), p. 59. Black, D. C. 1972, Geochim. Cosmochim. Acta, 36, 347

1983, Ap. J., 266, 889.

Breneman, H. H and Stone, E. C. 1985, Ap. J.(Letters), 299, L57 (B\&S).

Cameron, A. G. W. 1982, in Essays in Nuclear Astrophysics, ed. C. A. Barnes, D. D. Clayton, and D. N. Schramm (Cambridge: Cambridge University Press).

Dietrich, W. F., and Simpson, J. A. 1979, Ap. J. (Letters), 231, L91. 1981, Ap. J. (Letters), 245, L41.

Geiss, J., Buehler, F., Cerutti, H., Eberhardt, P., and Filleux, Ch. 1972, Apollo 16 Preliminary Science Report (NASA SP-315), p. 14-1.

Luhn, A., Klecker, B., Hovestadt, D., Gloeckler, G., Ipavich, F. M., Scholer, M., Fan, C. Y., and Fisk, L. A. 1984, Adv. Space Res., 4 (Nos. 2-3), 161.

\section{REFERENCES}

Mason, G. M., Fisk, L. A., Hovestadt, D., and Gloeckler, G. 1980, Ap. J., 239, 1070.

McGuire, R. E., von Rosenvinge, T. T., and McDonald, F. B. 1979, Proc. 16th Internat. Cosmic Ray Conf. (Kyoto), 5, 61 .

Mewaldt, R. A. 1980, in The Ancient Sun, ed. R. O. Pepin, J. A. Pepin, and R. B. Merrill (New York: Pergamon), p. 81.

Mewaldt, R. A., Spalding, J. D., and Stone, E. C. 1984, Ap. J., 280, 892.

Mewaldt, R. A., Spalding, J. D., Stone, E. C., and Vogt, R. E. 1979, Ap. J. (Letters), 231, L97.

Podosek, F. A. 1978, Ann. Rev. Astr. Ap., 16, 293.

Simpson, J. A., Wefel, J. P., and Zamow, R. 1983, Proc. 18th Internat. Cosmic Ray Conf. (Bangalore), 10,332.

Wieler, R., Baur, H., and Signer, P. 1986, Geochim. Cosmochim. Acta, 50, 1997.

R. A. Mewaldt and E. C. Stone: 220-47 Downs Laboratory of Physics, California Institute of Technology, Pasadena, CA 91125 